\title{
SiRNA-Expressing SV40
}

National Cancer Institute

\section{Source}

National Cancer Institute. siRNA-Expressing SV40. NCI Thesaurus. Code C62536.

A simian virus 40 (SV40)-based shuttle vector, encoding small interfering RNA (siRNA), with potential antineoplastic activity. The expression of siRNA in target tumor cells transfected with an siRNA-expressing SV40 vector may result in siRNA-mediated silencing of target oncogenes and, so, the inhibition of tumor cell growth and the induction of tumor cell death. 